\title{
MicroRNA-155 inhibits migration of trophoblast cells and contributes to the pathogenesis of severe preeclampsia by regulating endothelial nitric oxide synthase
}

\author{
XUELAN LI, CHUNFANG LI, XIN DONG and WENLI GOU \\ Department of Obstetrics and Gynaecology, The First Affiliated Hospital, \\ Xi'an Jiao Tong University, Xi'an, Shaanxi 710061, P.R. China
}

Received September 29, 2013; Accepted March 21, 2014

DOI: $10.3892 / \mathrm{mmr} .2014 .2214$

\begin{abstract}
The aim of the present study was to characterize the role of microRNA (miR)-155 in the pathogenesis of severe preeclampsia (PE). A total of 19 severe preeclampsic and 22 normal placentas were collected to measure miR-155 and endothelial nitric oxide synthase (eNOS) expression using quantitative (q)PCR and western blot analysis. The results demonstrated a significant increase in the levels of miR-155 and decreased eNOS expression in the severe preeclampsic placentas, as compared with the normal controls. In order to examine the function of miR-155 in the human placenta, the HTR8/Svneo cell line was transiently transfected with an miR-155 mimic or its inhibitor, anti-miR-155. It was confirmed that miR-155 may suppress the expression of eNOS in HTR-8/SVneo cells. Furthermore, a transwell insert invasion assay demonstrated that miR-155 inhibited cell invasion in trophoblast cells, and the effect was rescued by over expression of eNOS. The present study revealed that miR-155 has a negative regulatory role in the migratory behavior of HTR-8/SVneo cells via modulating eNOS.
\end{abstract}

\section{Introduction}

Preeclampsia (PE) is a medical condition characterized by hypertension and proteinuria in pregnant females following 20 weeks of gestation. It is the most common cause of fetal morbidity and mortality, which has no curative therapeutic strategy, except for delivery of the placenta (1). The association between PE and aberrant microRNA (miRNA) expression in placentas was first reported in 2007 (2) and a number of other similar findings have since been demonstrated (3). Considering the previously reported role of miRNAs in the development

Correspondence to: Dr Wenli Gou, Department of Obstetrics and Gynaecology, The First Affiliated Hospital, Xi'an Jiao Tong University, 277 Yanta West Road, Xi'an, Shaanxi 710061, P.R. China E-mail: wenli_gou008@163.com

Key words: microRNA-155, endothelial nitric oxide synthase, preeclampsia of the placenta (4) and the generally accepted opinion that the abnormal development of the placenta at an early stage of gestation initiates this disease, it is important to elucidate the role of miRNA in the development of PE which may facilitate the understanding of the pathogenesis of the disease.

miRNA, a class of 22 -nucleotide-long non-protein coding RNAs, are able to regulate gene expression by binding to the 3' untranslated region (UTR) of target gene messenger RNA (mRNA), resulting in translational repression and/or mRNA degradation (5). It is generally considered that $\sim 1 / 3$ of all human genes may be regulated by miRNA (6), and that microRNA has a key role in cellular activities, including cell proliferation, apoptosis and immune responses (7-9). Therefore, understanding the regulatory network of miRNAs has attracted noteable attention in recent years.

Investigating the expression of miRNA and endothelial nitric oxide synthase (eNOS) in PE is important for the following reasons: i) Aberrant expression of eNOS may reduce the migratory capabilities of trophoblast cells (10) and the migratory capability is the key for deep placentation, the compromise of which may result in a wide spectrum of obstetric diseases, including PE (11); ii) ENOS may directly exacerbate the PE-like phenotype by interfering with the endothelin system (12); iii) functional polymorphisms in eNOS that alter enzymatic activity in vivo were reported to be associated with an increased risk for PE (13); iv) miR-155 was demonstrated to be an essential regulator of eNOS expression and endothelium-dependent vasorelaxation, and inhibition of miR-155 may restore eNOS expression and improve endothelial dysfunction $(14,15)$.

Based on the aforementioned evidence, it was hypothesized that miR-155 may function as a regulator of trophoblast cell behavior by modulating eNOS. To examine this hypothesis, the effect of miR-155 on the proliferation and invasion of trophoblast cells as well as the rescue effect of eNOS was investigated. The expression pattern of miR-155 and eNOS in preeclampsic placentas in comparison with the normal controls was also examined.

\section{Materials and methods}

Sample collection. The present study was performed with placenta tissues collected from 22 normal pregnant females 
and 19 severe PE patients at The First Affiliated Hospital, (Xi'an Jiao Tong University, Xi'An, Shaanxi, China). The study was approved by the Research Ethics Committees of the hospital and written informed consent were obtained from all of the subjects. Normal pregnancy was defined as a previously and currently normotensive female during pregnancy who delivered a healthy neonate following 37 weeks of gestation. Severe $\mathrm{PE}$ was define as a female patient without a history of hypertension presenting with systolic blood pressure $\geq 160 \mathrm{mmHg}$ or diastolic blood pressure $\geq 110 \mathrm{mmHg}$ on at least two occasions, combined with significant proteinuria following 20 weeks of gestation. The pregnant females who had renal disease, spontaneous abortion, gestational diabetes, fetal chromosomal or congenital abnormalities were excluded from the present study.

RNA extraction and quantitative polymerase chain reaction ( $q P C R)$. Total RNA was isolated from the cultured cells or placenta tissues using TRIzol reagent (Invitrogen Life Technologies, Carlsbad, CA, USA) following the manufacturer's instructions with few modifications. Reverse transcription and qPCR were conducted using the primer set: 5'-CTGTTAATGCTAATCGTGATAG-3' and 5'-GCAGGGTCCGAGGT-3' for miR-155 detection; primer set: 5'-CTCGCTTCGGCAGCACA-3' and 5'-AACGCTTCACGAATTTGCG T-3' for U6 detection; primer set: 5'-CCCTTCAGTGGCTGGTACAT-3' and 5'-CACGATGGTGACTTTGGCTA-3' for eNOS detection, and the SYBR Green real time detection system (Bio-Rad, Hercules, CA, USA), respectively. U6, an internal control, was used to normalize miR-155 levels.

Western blot analysis. Cell lysates were loaded onto an SDS-polyacrylamide gel for electrophoresis and then the proteins were transferred onto a polyvinylidene fluoride membrane (Beyotime Institute of Biotechnology, Haimen, China), which was blocked with TBST (10 mM Tris-Cl pH 8.0, $150 \mathrm{mM} \mathrm{NaCl}$ and $0.05 \%$ Tween-20) containing 5\% non-fat dry milk powder at room temperature for $1 \mathrm{~h}$. The membrane was then incubated with the rabbit anti-eNOS polyclonal antibody (1:1500; Abcam, Cambridge, UK) at $4^{\circ} \mathrm{C}$ overnight, followed by incubation with horseradish peroxidase-anti-rabbit secondary antibody (Abcam) at room temperature for $1 \mathrm{~h}$. Chemical fluorescence was detected using an enhanced chemiluminescence kit (Amersham Biosciences, Piscataway, NJ, USA) according to the manufacturer's instructions. The target bands were densitometrically analyzed and normalized by $\beta$-actin.

Cell lines and cell culture. The immortalized trophoblast cell line, HTR8/Svneo, was purchased from the American Type Culture Collection (ATCC; Manassas, VA, USA). Cells were cultured in RPMI-1640 (Invitrogen Life Technologies) containing $10 \%$ fetal bovine serum (Sigma-Aldrich Canada Co., Oakville, ON, Canada), $100 \mathrm{U} / \mathrm{ml}$ penicillin and $100 \mathrm{mg} / \mathrm{ml}$ streptomycin (Invitrogen Life Technologies).

Plasmids and transfection. The small interfering (si)RNA, duplex against human eNOS (anti-eNOS-siRNA), the mimics for miR-155, the miR-155 inhibitor (anti-miR-155) and the scramble control were purchased from Ambion, Inc. (Foster City, CA, USA). The coding sequence for eNOS was ampli- fied and inserted into a pcDNA4.0 vector (Invitrogen Life Technologies) to construct the eNOS expression plasmid (pcDNA4-eNOS).

Cell survival and proliferation assay. Untreated or pre-treated cells were cultured in serum-free RPMI-1640 medium for three days. To examine the viability, cells were incubated in RPMI-1640 medium supplemented with $10 \%$ fetal bovine serum in 6-well plates for $48 \mathrm{~h}$ and the cell number was counted using the trypan blue exclusion method. Dead cells were defined as those stained with trypan blue dye. The percentage of living cells was calculated by the association between the number of viable cells and the total number of cells counted.

Transwell invasion and migration assay. A transwell insert invasion assay was performed in 24 well fitted inserts with membranes (pore size, $8 \mathrm{~mm}$; Costar, Cambridge, MA, USA). As described previously (16), cell invasion was examined using a polycarbonate membrane cell culture insert (Costar, Corning Inc., Corning, NY, USA) coated with growth factor reduced Matrigel (BD Biosciences, Bedford, MA, USA). The cells transfected with the control, miR-155 mimic, anti-miR-155 or eNOS, were treated with $10 \mathrm{mg} / \mathrm{ml}$ mitomycinC (SigmaAldrich, St. Louis, MO, USA) for $2 \mathrm{~h}$ and placed on top of the wells at a density of $2.5 \times 10^{5}$ cells/well. For the 'rescue experiment', pDNA4-eNOS or its control plasmid was transfected into the cells, respectively. Invaded cells on the lower surface of the membrane were stained with crystal violet and counted.

Statistical analysis. The results of the transwell insert invasion assay, proliferation assay, qPCR, western blotting, densitometry analysis and clinical characteristics of the severe PE group and control group, were performed in triplicate and expressed as the mean \pm standard error of the mean. Student's t-test or one-way analysis of variance was used to analyze the differences. The statistical analysis was conducted by SPSS 17.0 software (SPSS, Inc., Chicago, IL, USA). $\mathrm{P}<0.05$ was considered to indicate a statistically significant difference.

\section{Results}

Expression levels of miR-155 and eNOS in preeclampsic placentas. The expression levels of miR-155 and eNOS in the placentas were measured and compared between the two groups comprising of 19 patients with severe PE and 22 healthy pregnant females. No significant differences were identified between the normal pregnant and the preeclamptic female patients with respect to maternal age, body mass index, glucose tolerance, infant birth weight and gestational age, as summarized in Table I. The results demonstrated that the relative expression of miR-155 in preeclampsic placentas was significantly upregulated to $\sim 260 \%$ compared with the normal placentas (Fig. 1A). The eNOS protein level was also measured using western blot analysis and the relative density of eNOS in the placentas from severe PE patients was $\sim 4$-fold more than that of the controls (Fig. 1B). The ENOS mRNA expression level was also measured by qPCR and it was identified that the mRNA level in the placentas derived from severe preeclampsic female patients was significantly lower than the normal placentas (Fig. 1C). 
Table I. Distribution of the selected variables between the severe PE cases and control subjects.

\begin{tabular}{lccr}
\hline Variable & Control $(\mathrm{n}=19)$ & Severe PE $(\mathrm{n}=22)$ & P-value \\
\hline Maternal age (years) & $28.2 \pm 4.5$ & $27.6 \pm 4.2$ & 0.661 \\
Body mass index $\left(\mathrm{kg} / \mathrm{m}^{2}\right)$ & $26.2 \pm 3.2$ & $26.8 \pm 3.4$ & 0.566 \\
Systolic BP (mmHg) & $115 \pm 18$ & $171 \pm 8.2$ & $<0.001$ \\
Diastolic BP (mmHg) & $81 \pm 12$ & $109 \pm 9.3$ & $<0.001$ \\
Gestational age (weeks) & $38.5 \pm 2.3$ & $37.4 \pm 2.1$ & 0.117 \\
50 GCT (mmol/l) & $7.1 \pm 1.1$ & $7.0 \pm 0.9$ & 0.750 \\
24 h urine protein $(\mathrm{g})$ & $0.02 \pm 0.04$ & $4.21 \pm 2.12$ & $<0.001$ \\
Infant birth weight $(\mathrm{g})$ & $3460 \pm 220$ & $3350 \pm 208$ & 0.108 \\
Placenta weight $(\mathrm{g})$ & $521 \pm 69$ & $502 \pm 55$ & 0.332 \\
\end{tabular}

PE, preeclampsia; BP, blood pressure; GCT, glucose challenge test.

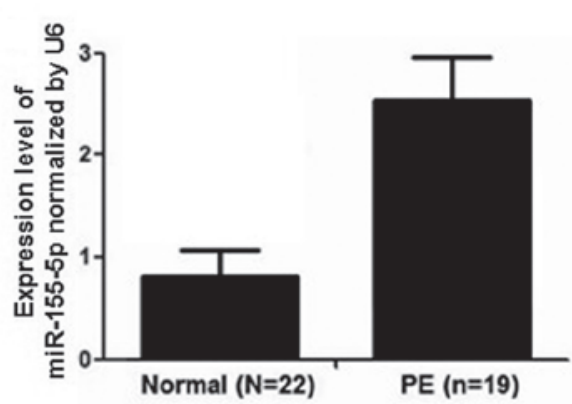

C

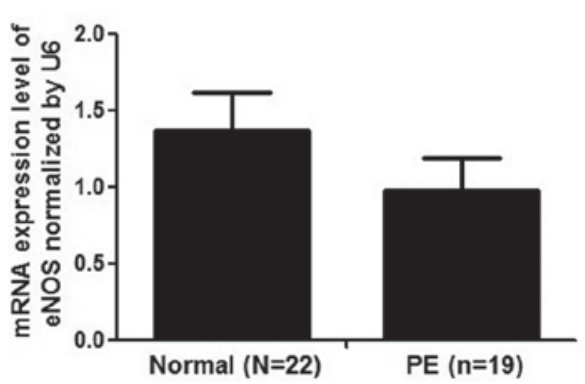

B
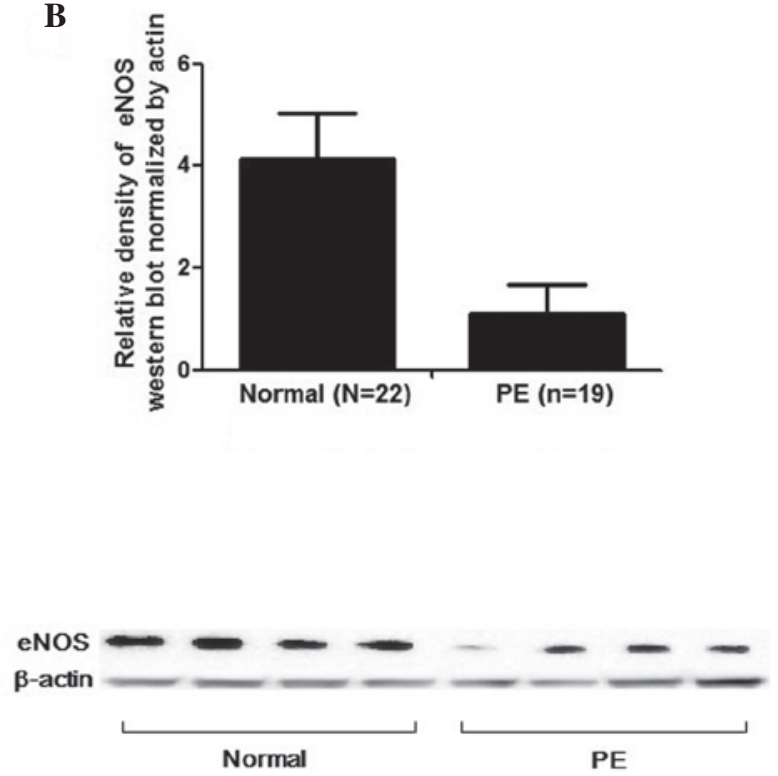

Figure 1. Comparison of the expression patterns of miR-155 and eNOS between preeclampsic and normal placentas. (A) The expression of miR-155-5p in 19 preeclamptic placentas (PE) and 22 normal placentas (Control). (B) Western blot analysis demonstrating the protein levels of eNOS in placentas from 19 PE and 22 Control (left bar chart, densitometry analysis and statistical comparison; right panel, selected results of western blot, four samples/group). (C) The mRNA expression of eNOS in placentas from 19 PE and 22 Control. PE, preeclampsia; eNOS, endothelial nitric oxide synthase; miR, microRNA.

Effect of miR-155 on the invasion and proliferation of trophoblast cells. The invasion and cell proliferation of HTR8/SVneo cells were examined in order to further investigate the effect of miR-155 on trophoblast cell behavior. First, the inhibitory effect of miR-155 on the mRNA and protein expression levels of eNOS was confirmed. As demonstrated in Fig. 2A, the inhibitory effect of specific anti-eNOS siRNA was $\sim 2 \mathrm{X}$ higher than that of the miR-155. Secondly, it was identified that neither upregulation nor downregulation of miR-155 in HTR8/SVneo cells affected the cell viability (data not shown). Furthermore, a transwell insert invasion assay was performed with the cells treated with mitomycin $\mathrm{C}$ that was used to eliminate the possible effect of cell growth on cell invasion. The results revealed that inhibition of miR-155 in HTR8/SVneo cells evidently promoted cell invasiveness (Fig. 3A and B).
Consistently, the overexpression of miR-155 significantly suppressed cell invasiveness and the inhibitory effect of miR-155 was $2 \mathrm{X}$ higher than that of the anti-eNOS-siRNA (Fig. 2B).

Effect of overexpression of eNOS on miR-155 regulated cellular behavior. To identify whether eNOS was directly involved in the miR-155 invasion-regulating effect, eNOS (pcDNA4-ENOS) was overexpressed in the cells transfected with miR-155 or anti-eNOS-siRNA, respectively, to achieve a 'rescue' effect. The results demonstrated that the overexpression of eNOS completely blocked the effect of anti-eNOS-siRNA in HTR8/SVneo cells. However it only restored $80 \%$ of the migratory capability in the cells transfected with miR-155 (Fig. 2B). 
A

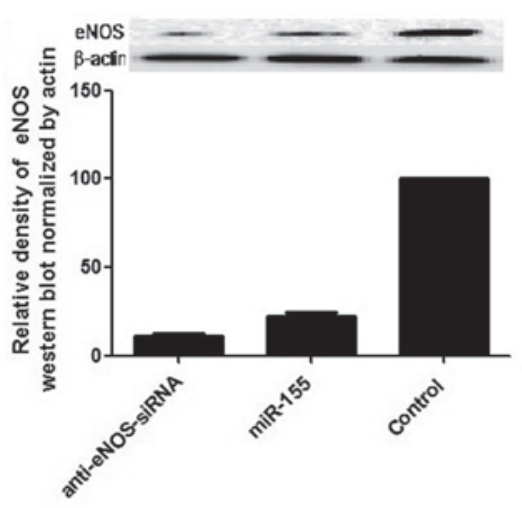

B

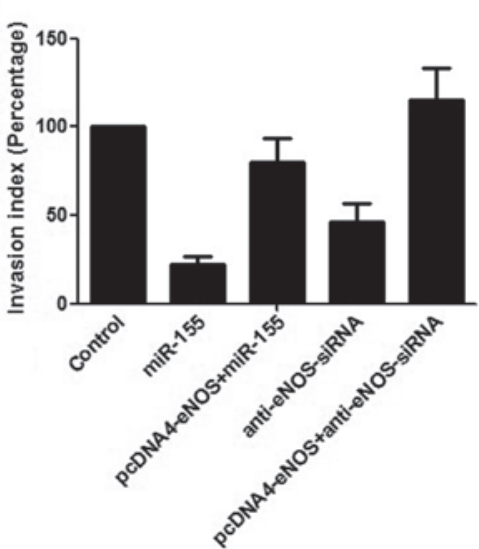

Figure 2. Effect of miR-155 on the upregulation of cell invasion in HTR8/SVneo cells. (A) Suppression of eNOS by miR-155 mimic and anti-eNOS siRNA in HTR8/SVneo cells (upper panel, selected results of the western blot; lower bar chart, densitometry analysis and statistical comparison). (B) A transwell insert assay was utilized to examine cell invasiveness by transfecting HTR8/SVneo cells with the control, miR-155 (miR-155 mimic), siRNA for eNOS (anti-eNOS siRNA), and the 'rescue' effect by overexpressing eNOS in HTR8/SVneo cells transfected with miR-155 mimic and anti-eNOS siRNA, respectively. miRNA, microRNA; PE, preeclampsia; eNOS, endothelial nitric oxide synthase; siRNA, small interfering RNA.

A

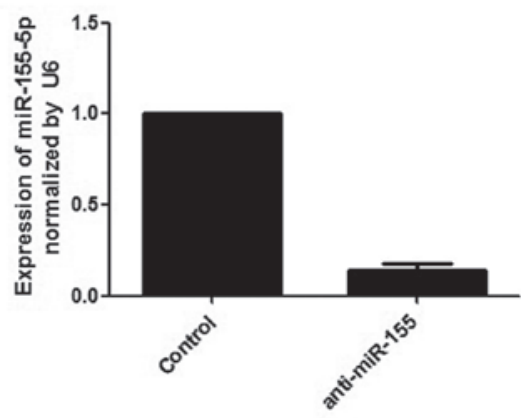

B

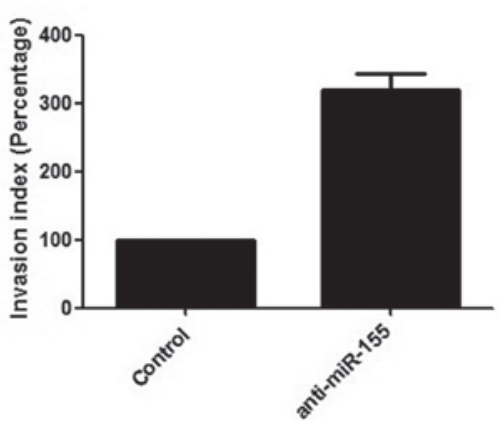

Figure 3. Effect of miR-155-5p downregulation on cell invasion in HTR8/SVneo cells. (A) Suppression of miR-155-5p by its inhibitor, anti-miR-155-5p in HTR8/SVneo cells. (B) A transwell insert assay was utilized to examine cell invasiveness transfected with the inhibitor control or anti-miR-155. The value of invasion index in the corresponding $\mathrm{NC}$ was set as 100. miRNA, microRNA; NC, negative control.

\section{Discussion}

miRNA is considered to have an important role in gene expression regulation and is known to contribute to the development of numerous diseases. Key components of the miRNA biosynthesis pathway have been identified in placenta tissues, and aberrant expression of microRNAs and their target genes has been detected in the placentas obtained from preeclampsic pregnancies (17-20), indicating a significant role for microRNAs in regulating placental development, function and disease pathogenesis. The mechanisms underlying how miRNAs modulate trophoblast cell function and placental development, however, remains mostly elusive.

Genetic components have been considered to have an important role in the development of PE (21). Previous linkage analyses have located the PE susceptibility locus to chromosome $7 \mathrm{q} 35$ to 36 and have identified eNOS as one of the susceptibility genes of PE $(22,23)$. A common polymorphism of the eNOS gene that enhances the degradation of the enzyme, results in an aberrantly low production of nitric oxide (NO) as well as an increased risk for PE in the carriers of the rare allele (24-26). In one study, eNOS knockout mice presented with symptoms, including hypertension, insulin resistance, hyperlipidemia and decreased production of NO (27). In addition, the expression of eNOS was evidently decreased in the umbilical vessel of female patients with PE (28) and reduced maternal eNOS/nitric oxide exacerbated the PE-like phenotype through activation of the endothelin system (12). This evidence indicates that eNOS is important in the pathogenesis of PE. Simultaneously, miR-155 was identified to be an essential regulator of eNOS expression and endothelium-dependent vasorelaxation, and it was identified that inhibition of miR-155 may restore eNOS expression and improve endothelial dysfunction $(14,15)$. In the present study, it was confirmed that miR-155 suppresses the expression of eNOS in trophoblast cells and the expression pattern of miR-155 and eNOS in PE and normal placentas was demonstrated. As expected, a significantly higher level of miR-155 and lower level of eNOS expression were detected in the 19 preeclampsic placentas compared with the 22 normal placentas.

The invasion of trophoblast cells into the endometrial stroma and inner third of the myometrium is a crucial step for the development of the maternal-fetal circulation. Compromised migration of trophoblast cells may cause insufficient placentation, which has been reported to be associated with various pathological processes, including fetal growth retardation, PE and spontaneous abortion (11). miR-155 has 
been reported to be involved in the regulation of the migratory capability of trophoblast cells through more than one pathway. Dai et al reported that cyclin D1 promoted migration of trophoblast cells and the suppression of cyclin D1 by miR-155 decreased the migratory ability of the cells, mediated by the phosphorylation of FLNa (25). Similarly, miR-155 may also affect the occurrence of PE by affecting trophoblast migration via downregulating CYR61 (26). In the present study, it was demonstrated that miR-155 inhibited trophoblast cell migration and invasion via regulating eNOS. In order to examine the function of miR-155 in the human placenta, miR-155 mimic or its inhibitor were transfected into HTR8/Svneo cells. miR-155 was identified to possess a strong inhibitory effect on trophoblast cell migration and invasion. Consistently, the suppression of miR-155 by anti-miR-155, led to promotion of cell migration and invasion, indicating that endogenous miR-155 significantly contributed to the regulation of this cellular behavior. Notably, the silencing effect of anti-eNOS-siRNA was $\sim 2 \mathrm{X}$ higher than the miR-155 mimic, whereas the inhibitory effect of miR-155 on trophoblast cell migration was paradoxically more potent than anti-eNOS-siRNA. It was reasoned that miR-155 may suppress the migration of the cell by downregulating other components, including cyclin D1 (25) or CYR61 (26). This hypothesis was supported by the results of the 'rescue' experiment, where eNOS was cloned into a pcDNA4 vector and overexpressed in the HTR-8/SVneo cells transfected with the miR-155 mimic or anti-eNOS-siRNA. As demonstrated in Fig. 2, the suppression of migration was completely restored in the anti-eNOS-siRNA transfected cells by overexpressing eNOS. By contrast, in miR-155 mimic transfected cells, eNOS only restored $80 \%$ of the migratory capability of the intact cells, which was still higher than expected. It was reasoned that the messenger, such as cGMP, may partially compensate for the lost signal of CYR61 if eNOS is overexpressed (29).

In conclusion, the present study has revealed a negative regulatory role of miR-155 in the migration of HTR-8/SVneo cells via modulating eNOS. eNOS is confirmed as a target of miR-155 in trophoblast cells and miR-155 is identified as a key regulator in the development of severe $\mathrm{PE}$. These data provide insight into the molecular mechanisms of diseases associated with trophoblasts $2 \mathrm{x}$ higher and may facilitate in further elucidating the pathogenic role of miR-155 in PE.

\section{References}

1. Redman CW: Current topic: pre-eclampsia and the placenta Placenta 12: 301-308, 1991.

2. Pineles BL, Romero R, Montenegro D, et al: Distinct subsets of microRNAs are expressed differentially in the human placentas of patients with preeclampsia. Am J Obstet Gynecol 196: 261, 2007.

3. Zhu XM, Han T, Sargent IL, Yin GW and Yao YQ: Differential expression profile of microRNAs in human placentas from preeclamptic pregnancies vs normal pregnancies. Am J Obstet Gynecol 200: 661, 2009.

4. Fu G, Brkić J, Hayder $\mathrm{H}$ and Peng C: MicroRNAs in human placental development and pregnancy complications. Int J Mol Sci 14: 5519-5544, 2013.

5. Lewis BP, Burge CB and Bartel DP: Conserved seed pairing, often flanked by adenosines, indicates that thousands of human genes are microRNA targets. Cell 120: 15-20, 2005.

6. Yu J, Wang F, Yang GH, Wang FL, Ma YN, et al: Human microRNA clusters: genomic organization and expression profile in leukemia cell lines. Biochem Biophys Res Commun 349: 59-68, 2006
7. Brennecke J, Hipfner DR, Stark A, Russell RB and Cohen SM: Bantam encodes a developmentally regulated microRNA that controls cell proliferation and regulates the proapoptotic gene hid in Drosophila. Cell 113: 25-36, 2003.

8. Calame K: MicroRNA-155 function in B Cells. Immunity 27: 825-827, 2007.

9. Jopling CL, Yi M, Lancaster AM, Lemon SM and Sarnow P: Modulation of hepatitis $\mathrm{C}$ virus RNA abundance by a liver-specific microRNA. Science 309: 1577-1581, 2005

10. Corthorn J, Germain AA, Chacón C, et al: Expression of kallikrein, bradykinin b2 receptor, and endothelial nitric oxide synthase in placenta in normal gestation, preeclampsia, and placenta accreta. Endocrine 29: 491-499, 2006.

11. Zhu JY, Pang ZJ and Yu YH: Regulation of trophoblast invasion: the role of matrix metalloproteinases. Rev Obstet Gynecol 5: e137-e143, 2012.

12. Li F, Hagaman JR, Kim HS, et al: eNOS deficiency acts through endothelin to aggravate sFlt-1-induced pre-eclampsia-like phenotype. J Am Soc Nephrol 23: 652-660, 2012.

13. Yu CK, Casas JP, Savvidou MD, Sahemey MK, Nicolaides KH and Hingorani AD: Endothelial nitric oxide synthase gene polymorphism (Glu298Asp) and development of pre-eclampsia: a case-control study and a meta-analysis. BMC Pregnancy Childbirth 6: 7, 2006.

14. Zhang XG, Hong Q, Hou K, Wang YD, Wu D and Chen XM: High concentration uric acid regulates endothelial function via miR-155. Nan Fang Yi Ke Da Xue Xue Bao 33: 1141-1145, 2013 (In Chinese).

15. Sun HX, Zeng DY, Li RT, et al: Essential role of microRNA-155 in regulating endothelium-dependent vasorelaxation by targeting endothelial nitric oxide synthase. Hypertension 60: 1407-1414, 2012.

16. Liu J, Maccalman CD, Wang YL and Leung PC: Promotion of human trophoblasts invasion by gonadotropin-releasing hormone (GnRH) I and GnRH II via distinct signaling pathways. Mol Endocrinol 23: 1014-1021, 2009.

17. Donker RB, Mouillet JF, Nelson DM and Sadovsky Y: The expression of Argonaute2 and related microRNA biogenesis proteins in normal and hypoxic trophoblasts. Mol Hum Reprod 13: 273-279, 2007.

18. Luo SS, Ishibashi O, Ishikawa G, et al: Human villous trophoblasts express and secrete placenta-specific microRNAs into maternal circulation via exosomes. Biol Reprod 81: 717-729, 2009.

19. Enquobahrie DA, Abetew DF, Sorensen TK, Willoughby D, Chidambaram K and Williams MA: Placental microRNA expression in pregnancies complicated by preeclampsia. Am J Obstet Gynecol 204: 178, 2011.

20. Mayor-Lynn K, Toloubeydokhti T, Cruz AC and Chegini N: Expression profile of microRNAs and mRNAs in human placentas from pregnancies complicated by preeclampsia and preterm labor. Reprod Sci 18: 46-56, 2011.

21. Pridjian G and Puschett JB: Preeclampsia: Part 2: experimental and genetic considerations. Obstet Gynecol Surv 57: 619-640, 2002.

22. Guo G, Lade JA, Wilton AN, et al: Genetic susceptibility to preeclampsia and chromosome 7q36. Hum Genet 105: 641-647, 1999.

23. Lade JA, Moses EK, Guo G, et al: The eNOS gene: a candidate for the preeclampsia susceptibility locus? Hypertens Pregnancy 18: 81-93, 1999

24. Demirçubuk AG, Coşkun MY, Demiryürek Ş, et al: Endothelial NOS gene Glu298Asp polymorphism in preterm neonates with respiratory distress syndrome. Pediatr Pulmonol 48: 976-980, 2013.

25. Dai Y, Qiu Z, Diao Z, et al: MicroRNA-155 inhibits proliferation and migration of human extravillous trophoblast derived HTR-8/SVneo cells via down-regulating cyclin D1. Placenta 33: 824-829, 2012

26. Zhang Y, Diao Z, Su L, Sun H, Li R, Cui H and Hu Y: MicroRNA-155 contributes to preeclampsia by down-regulating CYR61. Am J Obstet Gynecol 202: 466, 2010.

27. Duplain H, Burcelin R, Sartori C, et al: Insulin resistance, hyperlipidemia, and hypertension in mice lacking endothelial nitric oxide synthase. Circulation 104: 342-345, 2001.

28. Xiang W, Chen H, Hu L and Xu X: Endothelial nitric oxide synthase traffic inducer in the umbilical vessels of the patients with pre-eclampsia. J Huazhong Univ Sci Technolog Med Sci 29: 243-245, 2009

29. Histing T, Marciniak K, Scheuer C, et al: Sildenafil accelerates fracture healing in mice. J Orthop Res 29: 867-873, 2011. 